\title{
Non-porous organic crystals and their interaction with guest molecules from the gas phase
}

\author{
Mirian Elizabeth Casco ${ }^{1}$ ( $\cdot$ Felix $\mathrm{Krupp}^{2} \cdot$ Sven Grätz $^{3} \cdot$ Alexander Schwenger $^{2} \cdot$ Vassiliki Damakoudi $^{2}$.

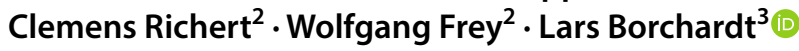

Received: 6 April 2020 / Revised: 16 July 2020 / Accepted: 25 August 2020 / Published online: 18 September 2020

(c) The Author(s) 2020

\begin{abstract}
Some organic molecules encapsulate solvents upon crystallization. One class of compounds that shows a high propensity to form such crystalline solvates are tetraaryladamantanes (TAAs). Recently, tetrakis(dialkoxyphenyl)-adamantanes have been shown to encapsulate a wide range of guest molecules in their crystals, and to stabilize the guest molecules against undesired reactions. The term 'encapsulating organic crystals' (EnOCs) has been coined for these species. In this work, we studied the behavior of three TAAs upon exposition to different guest molecules by means of sorption technique. We firstly measured the vapor adsorption/desorption isotherms with water, tetrahydrofuran and toluene, and secondly, we studied the uptake of methane on dry and wet TAAs. Uptake of methane beyond one molar equivalent was detected for wet crystals, even though the materials showed a lack of porosity. Thus far, such behavior, which we ascribe to methane hydrate formation, had been described for porous non-crystalline materials or crystals with detectable porosity, not for non-porous organic crystals. Our results show that TAA crystals have interesting properties beyond the formation of conventional solvates. Gas-containing
\end{abstract}

Mirian Elizabeth Casco and Felix Krupp contributed equally to this study.

Electronic supplementary material The online version of this article (https://doi.org/10.1007/s10450-020-00259-8) contains supplementary material, which is available to authorized users.

Extended author information available on the last page of the article 
organic crystals may find application as reservoirs for gases that are difficult to encapsulate or are slow to form crystalline hydrates in the absence of a host compound.

Wet tetraaryladamantane crystals take up methane in form of methane hydrate structure I, even though they appear nonporous to argon.

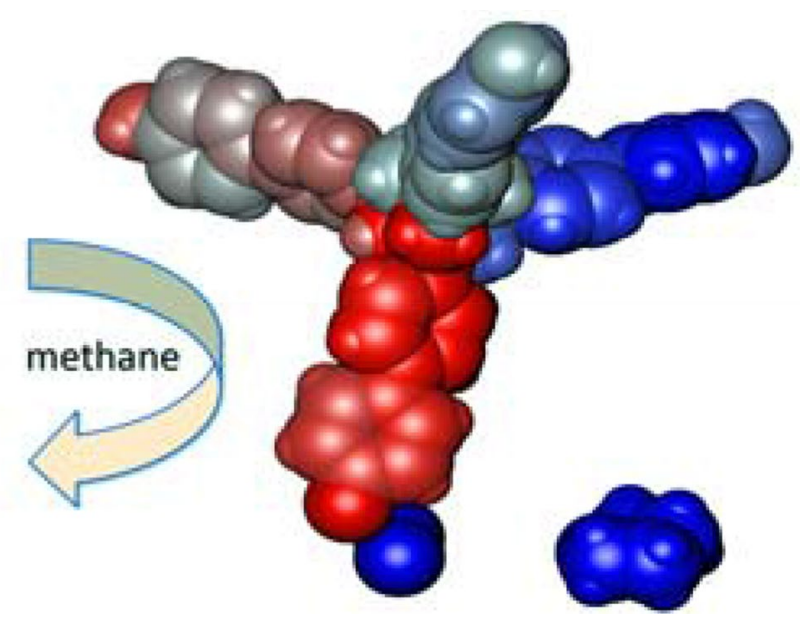

Keywords Adamantane $\cdot$ Adsorption isotherms $\cdot$ Methane hydrate

\section{Introduction}

Rationally designed organic molecular crystals are gaining more and more significance in the adsorption fields (e.g. gas storage and separation) (Thallapally et al. 2007; Enright et al. 2003), where traditionally nanoporous materials including carbons, silica, zeolites and, recently, covalentorganic framework (COFs) and metal-organic framework (MOFs) are protagonists (Schüth et al. 2002). Unlike COFs and MOFs, which contain stable and extended nanoporous networks synthesized from organic molecular precursors (Chaoui et al. 2017; Zhou et al. 2012), the direct assembly of discrete organic molecules into porous structures is challenging; because they tend to pack efficiently to maximize the attractive intermolecular contact and to minimize crystals lattice voids.

Organic crystals are infinite arrays of like molecules. Other components are usually excluded from the crystalline array during assembly. The inclusion of solvent molecules is common only when no dense packing is achieved by the crystallizing organic molecules alone (Desiraju and Vittal 2011). The crystal arrangement in organic crystals is usually non-porous, but some solvates release the encapsulated solvent molecules at low pressure or elevated temperature, producing porous materials with void spaces that are accessible to gases (Tian et al. 2012). It has been reported that small gas molecules (e.g. methane or carbon dioxide) (Sozzani et al. 2005) can diffuse through the crystals lattice and allocate in these cavities, which has arisen the questions within scientists whether these systems have a comparable ability to store gases like traditional nanoporous materials.

Cooper et al. classified molecules that take up gases in the crystalline state as intrinsically porous (containing permanent covalent cavities) or extrinsically porous (inefficient packing upon crystallization) (Desiraju and Vittal 2011; Tian et al. 2012; Holst et al. 2010). Recently, crystals of organic cage molecules were developed that form materials with extremely large surface areas upon crystallization (Schneider et al. 2012; Zhang et al. 2014). However, in rare cases, the uptake of gases into organic crystals occurs without apparent or latent porosity, a phenomenon called "porosity without pores" (Barbour 2006), which is probably caused by temporary structural changes during uptake (Tian et al. 2009). An example of this phenomenon is that of calix[4] arenes, which capture guest molecules by forming a stable and a novel solid framework only stable in the presence of the guest, i.e. an inclusion compound (Tian et al. 2012). These systems were investigated for gas uptake in detail by Atwood et al. (Atwood et al. 2002; Thallapally et al. 2005; Atwood et al. 2004) and Ripmeester et al. (Enright et al. 2003; Brouwer et al. 2008; Ananchenko et al. 2008).

One class of organic compounds accessible via a small number of synthetic steps that possesses interesting properties as storage material are tetraaryladamantanes (TAAs). The TAA for which a broad range of inclusion complexes in the crystalline state was first reported by us is 
1,3,5,7-tetrakis(2,4-dimethoxyphenyl)adamantane (TDA). This octaether was originally prepared as an intermediate in the synthesis of branched oligonucleotide hybrids (Singh et al. 2011; Schwenger et al. 2017), but was studied as a crystalline host when it was found to give solvates with a large number of structurally diverse organic liquids (Schwenger et al. 2015). Stable crystalline inclusion complexes with reagents as different in structure and polarity as acetyl chloride, piperidine, cyclohexyl isocyanide and trimethylsilyl chloride were obtained (Schwenger et al. 2016). The ethyl derivative 1,3,5,7-tetrakis(2,4-diethoxyphenyl)adamantane (TEO) was then found to accommodate up to 3.5 equivalents of guest molecules in its crystals and to capture and release liquid aromatic hydrocarbons via the gas phase (Alexandre et al. 2017). Recently, cyclopentadiene was shown to be stabilized against dimerization by inclusion in TDA crystals, extending the scope of the crystal-based "formulations" to self-reactive organic substrates (Krupp et al. 2018).

Because of the breadth of the effect found for TAA octaethers, we coined the term "encapsulating organic crystals" (EnOCs) for these inclusion compounds (Richert and Krupp 2017). Unlike crystals based on compounds that cannot form tightly packed crystalline arrangements (Tian et al. 2009), the host molecules of EnOCs are able to assemble into closely packed crystals with a density typical for organic compounds (Schwenger et al. 2016). For example, TDA has been found to crystallize in a solvate-free triclinic system with a density of $1.30 \mathrm{~g} / \mathrm{cm}^{3}$, whereas some solvates have a lower density, such as the monoclinic form with acetone guest molecules (density $1.28 \mathrm{~g} / \mathrm{cm}^{3}$ ). Further, the EnOCforming TAAs do not have hydrogen bond donor moieties that strongly favor a specific molecular arrangement in the crystals, as found for Dianin's compound (Dianin 1914; Baker et al. 1956). Rather, EnOC-forming TAAs appear to have the ability to adopt many different crystalline arrangements with similar energy. In the presence of a guest molecule, they often assemble into a different lattice than in its absence.

Because most gases are atoms or small molecules, with limited intrinsic binding energy, it was unclear whether gases can induce the formation of a specific EnOC lattices that accommodates them or not. If they did, the small molecules would have to compete successfully with the large molecules of the host during crystal growth. This would be difficult, as the larger host molecules could displace the small guest molecule to give an entropically favored, solvatefree, closely packed form. We were therefore not surprised when our initial attempts to capture gases with TAAs were unsuccessful. It seemed rather unlikely that a hypothetical 'gas solvate' form, such as the one shown schematically in Fig. 1b, would exist.

But, the storage of gases is an important technical challenge and was a strong motivation to test this unlikely (a)

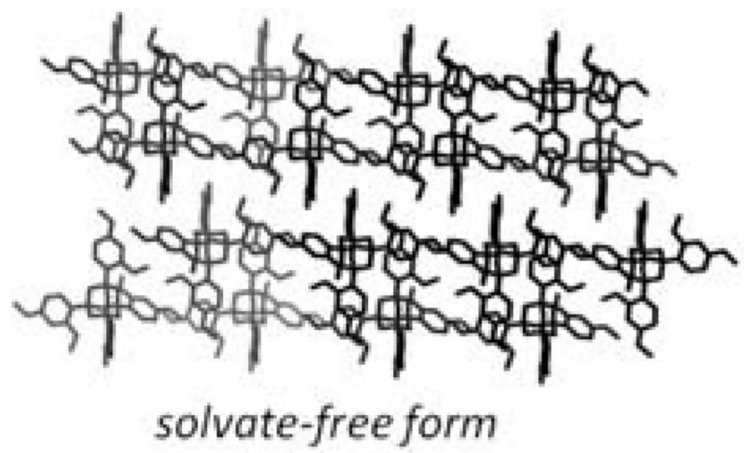

(b)

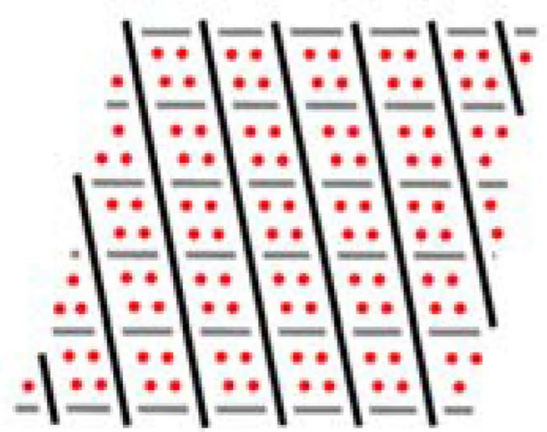

hypothetical 'gas solvate'

(c)

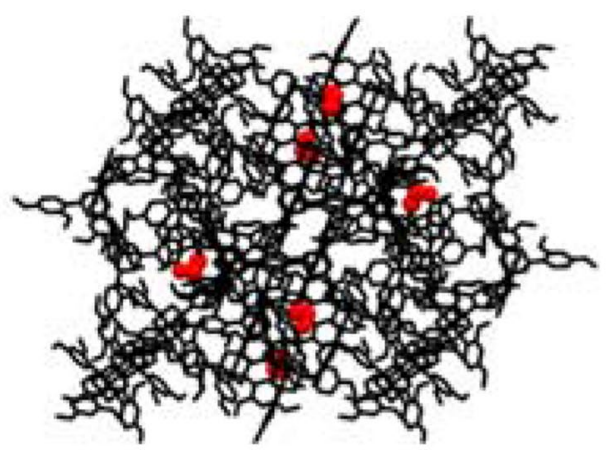

solvate

Fig. 1 Packing arrangements in different crystal structures of an EnOC-forming organic compound. a Known solvate-free form of TDA (Schwenger et al. 2015); b Cartoon of a hypothetical arrangement with gas molecules as guests in the crystalline lattice, and $\mathbf{c}$ known solvate form of TDA with a small solvent (ethanol) as guest molecule (Schwenger et al. 2015) (Color figure online)

hypotheses ( $\mathrm{Gu}$ et al. 2019). To achieve the capture, safe transport, and/or release of energy vectors, such as methane gas, requires new scientific and technological approaches. Here we report, firstly, on the uptake properties of TAA crystals toward different solvents from the gas phase: tetrahydrofuran, toluene and water. Secondly, we evaluate the ability of TAAs to uptake methane on their dry and wet crystals. 


\section{Results and discussion}

\subsection{Single-crystals of hosts}

For our study on gases as putative guest molecules in EnOCs, we chose the three compounds shown in Fig. 2. These encompass adamantanes with aryl arms of different size and structure, including adamantanes with dialkoxyphenyl arms (1 and 2) and 1,3,5,7-tetrakis( $p$-hydroxybiphenyl) adamantane (TBA, 3) with longer arms and terminal alcohol groups. The TAAs were prepared using known methods (Alexandre et al. 2017; Schwenger et al. 2015; Schilling et al. 2011; Singh et al. 2012), and were subjected to thermal crystallization to study their suitability for gas uptake.

For the single-crystal study, we focused on two solvents, namely THF as a more polar medium, and toluene as a more lipophilic crystallization medium. All three TAAs gave EnOCs with THF, whereas toluene was encapsulated in crystals of TDA and TEO only. Encapsulation of toluene in TBA was not successful because this TAA is too poorly soluble in this solvent. Figures 3 and 4 show molecular arrangements in single-crystal structures of the EnOCs obtained, and the results of these X-ray analyses are summarized in Table 1.

In case of TDA (1), monoclinic crystal systems were found for both solvents. The EnOC with THF also showed interesting details in the molecular arrangement (Fig. 3b). Besides the larger number of guest molecules (three molar equivalents), groups of THF molecules were in van der Waals contact, resulting in 'solvent pockets' and an extensive level of local solvation. Such solvate arrangements may facilitate structural dynamics and thus exchange processes.

Compound 2 (TEO) crystallized in a triclinic crystal system from either of the two solvents tested with a host/guest ratio of $1: 1$. Slight variations in the crystallization conditions led to the formation of two different inclusion complexes in case of TEO/THF (Fig. 3d, e). In the second crystal system, the host/guest ratio was unchanged at $1: 1$, but a monoclinic system had formed. This indicated that there are several crystal packings that are close in energy and that are both

(a)

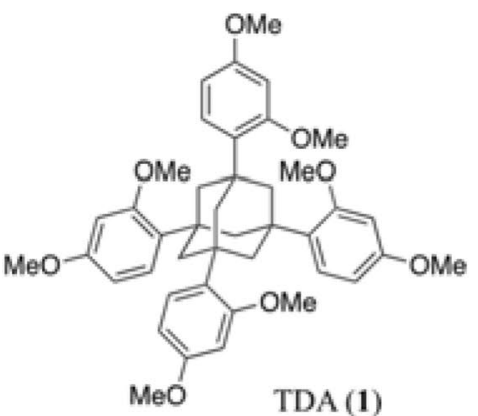

(b)

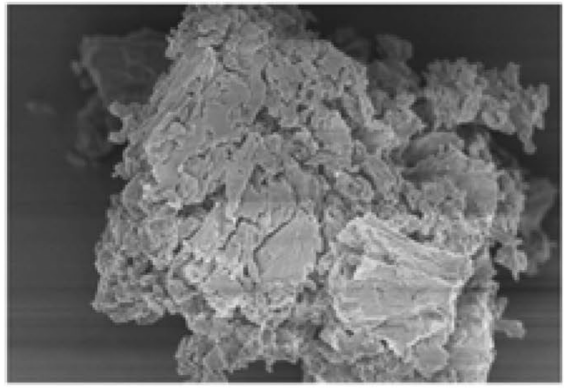

TDA

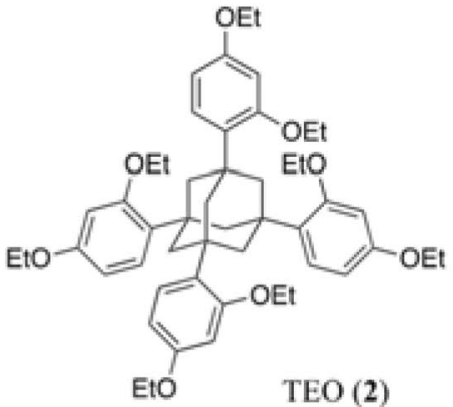

TEO (2)

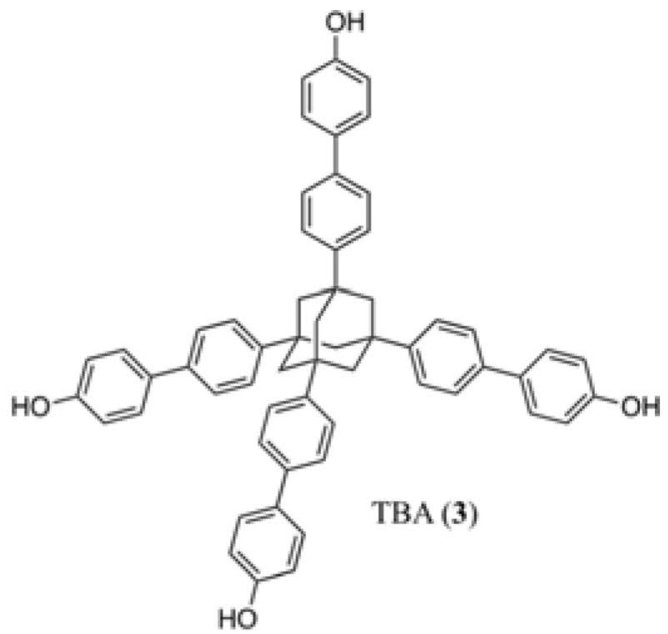

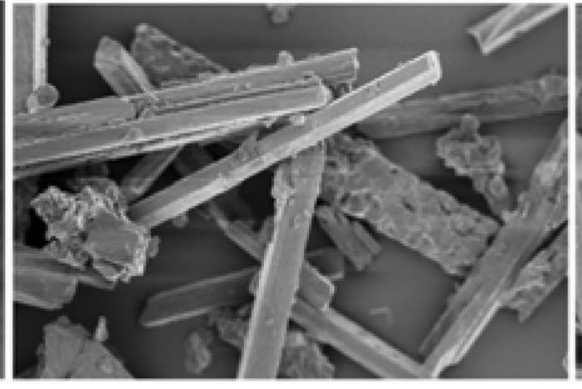

TEO

$50 \mu \mathrm{m}$

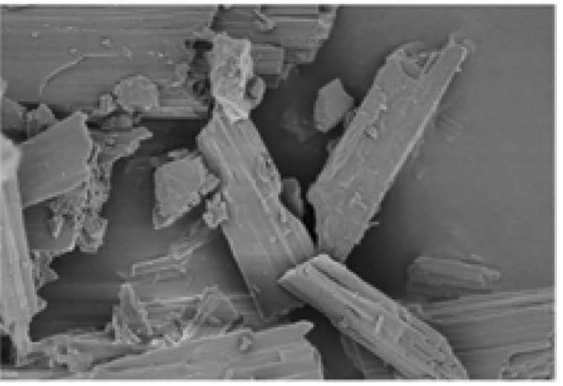

TBA

$10 \mu \mathrm{m}$

Fig. 2 Tetraaryladamantanes (TAAs) employed: a structures of 1,3,5,7-tetrakis(2,4-dimethoxyphenyl)adamantane (TDA, 1), 1,3,5,7-tetrakis(2,4-diethoxyphenyl)adamantane (TEO, 2), and 1,3,5,7-tetrakis( $p$-hydroxybiphenyl)adamantane (TBA, 3). b Scanning electron microscopy images of crystals of each of the materials after drying under vacuum $\left(1 \times 10^{-3} \mathrm{~Pa}\right)$ at $80^{\circ} \mathrm{C}$, prior to the gas sorption studies 
(a)

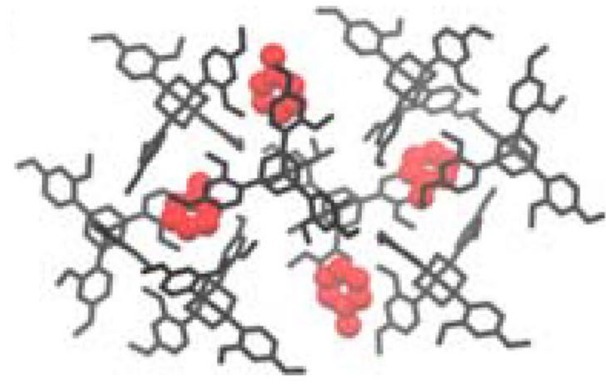

(b)

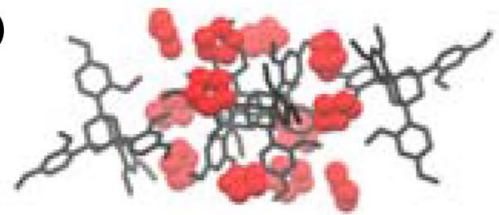

(c)

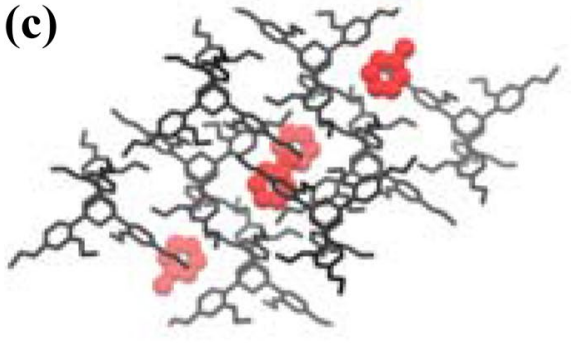

(d)

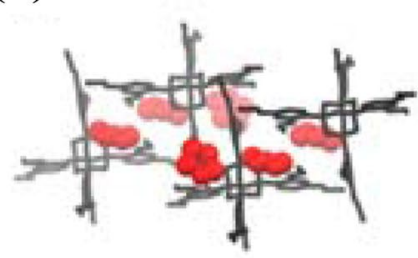

(e)

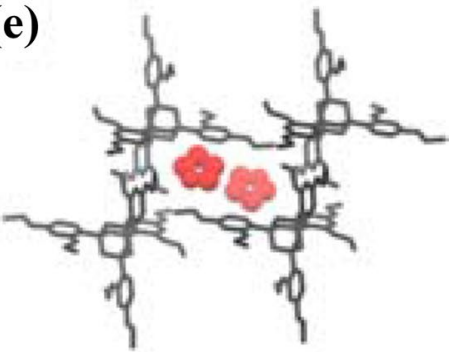

Fig. 3 Details of X-ray crystal structures of solvates of a TDA with toluene, $\mathbf{b}$ TDA with THF $\mathbf{c}$ TEO with toluene, $\mathbf{d}$ TEO with THF (triclinic crystal system) and e TEO with THF (monoclinic crystal system) molecules as guests (Color figure online)

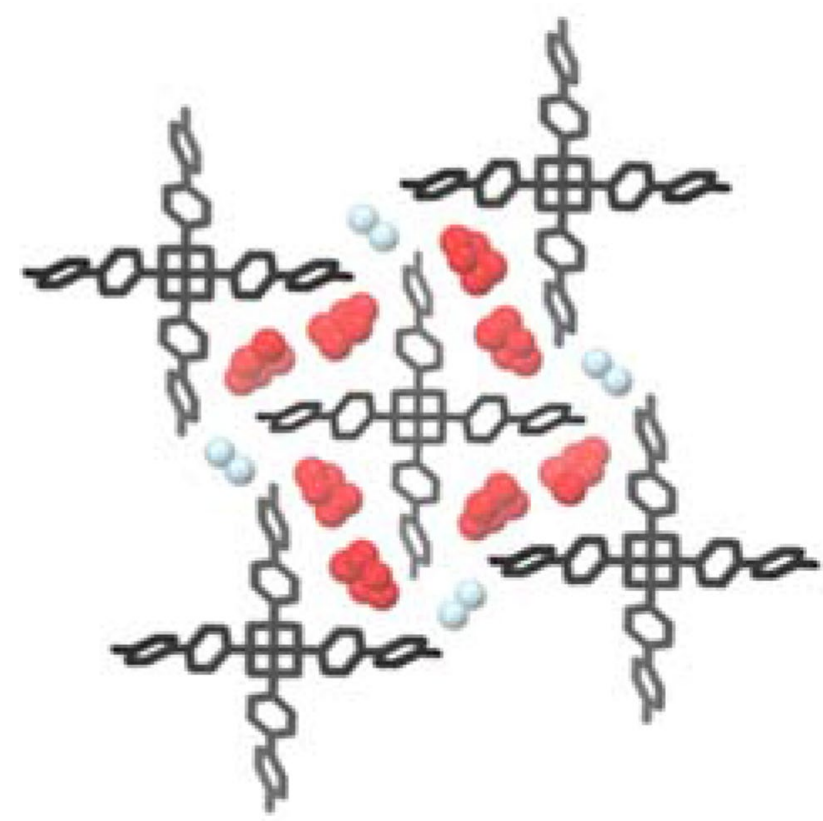

Fig. 4 X-ray crystal structure of a double solvate of TBA with THF (red) water molecules (light blue) as guests. The water molecules are disordered and every double sphere represents the two positions of one $\mathrm{H}_{2} \mathrm{O}$ entity (Color figure online) accessible kinetically. We assumed that this was a favorable trait for uptake and release processes that require changes in the molecular packing of the host.

In the case of TBA (3), there are hydroxyl groups at the 4'-position of the distal phenyl ring. This allows for hydrogen bonding with water molecules in the crystal. Figure 4 shows a portion of the X-ray crystal structure that was obtained from THF. A double solvate was found, i.e. two different solvent molecules reside in the unit cell, namely THF and water. The disordered water molecules engage in hydrogen bonding with $\mathbf{3}$ to give a cyclic network that is

Table 1 Structural parameters for inclusion complexes of TDA, TEO and TBA

\begin{tabular}{llllc}
\hline Host & Guest & Host/guest ratio & Crystal system & $\begin{array}{l}\text { Unit cell vol. } \\
\left(\AA^{3}\right)\end{array}$ \\
\hline TDA & Toluene & $2: 1$ & Monoclinic & 7528 \\
TDA & THF & $1: 3$ & Monoclinic & 4710 \\
TEO & Toluene & $1: 1$ & Triclinic & 2568 \\
TEO & THF & $1: 1$ & Triclinic & 2538 \\
TEO & THF & $1: 1$ & Monoclinic & 10,151 \\
TBA & THF/H $\mathrm{H}_{2} \mathrm{O}$ & $1: 4: 1$ & Tetragonal & 2976 \\
\hline
\end{tabular}

Obtained by crystallization from the organic guest compound as solvent. See the ESM for further details 
somewhat reminiscent of cavity-forming molecules, such as Dianin's compound (Dianin 1914; Baker at al. 1956). This hydrogen-bonded network leaves cavities filled with THF molecules as organic guests. The inclusion of THF occurs in overstoichiometric amounts, with a molar ratio of TBA/ $\mathrm{H}_{2} \mathrm{O} / \mathrm{THF}$ of 1:1:4 in the crystals. There are thus significant quantities of THF, but not infinite channels with sufficiently large diameters to allow for a free flow of molecules.

\subsection{Vapor/gas sorption}

Figure $2 b$ shows electron micrographs of TAA samples employed for the sorption study, confirming the crystallinity of the materials employed. Prior to the measurements, the crystals were dried under vacuum $\left(1 \times 10^{-3} \mathrm{~Pa}\right)$ at $80^{\circ} \mathrm{C}$ for $12 \mathrm{~h}$. Further details of the sorption study are given in the Electronic Supplementary Material (ESM). Physisorption of argon was measured at $87 \mathrm{~K}$ to evaluate the specific surface area of the three TAAs. Unlike nitrogen, the traditional probe molecule to characterize porosity, argon is a monoatomic molecule that has no dipole or quadrupole moment to interact with the surface of the adsorbent, making it more reliable. Figure 5 shows the results of the experiments.

According to the IUPAC classification, the shape of the isotherms is type III, typical for non-porous adsorbent (Thommes et al. 2015). This is expected for organic molecules with neither intrinsic nor extrinsic porosity. By applying the BET equation to the isotherm raw data, TEO and TBA gave specific surface areas smaller than $5 \mathrm{~m}^{2} / \mathrm{g}$, while TDA showed a value of $25 \mathrm{~m}^{2} / \mathrm{g}$. The argon uptake by TBA increased abruptly at relative pressures close to 1 . We attribute this to interparticle condensation that readily sets in at the chosen temperature for argon (Thommes et al. 2015).

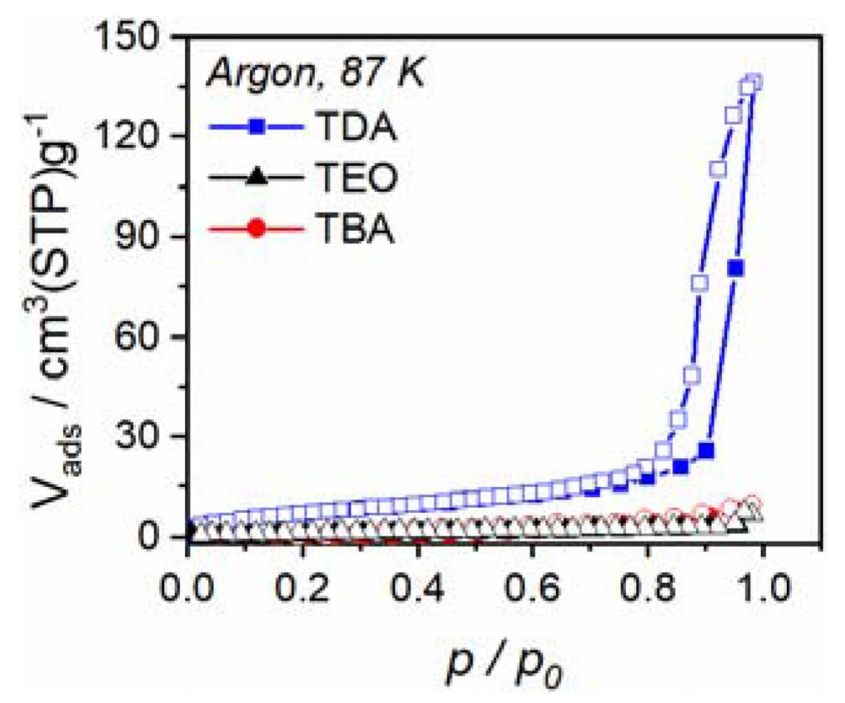

Fig. 5 Adsorption (closed symbols) and desorption (open symbols) isotherms for argon at $87 \mathrm{~K}$ with TAAs 1-3 (Color figure online)
Overall, this data shows that all three materials do not possess porosity, as uptake of argon (a gas showing very weak specific interactions with host structures) is insignificant, except for TDA in the interparticle condensation regime, close to a relative pressure of 1 at $87 \mathrm{~K}$.

Water adsorption/desorption measurements were then performed to test the response of the TAAs to a polar guest (Fig. 6). Both, TDA and TEO showed isotherms of type III, typical for non-porous materials, with weak adsorbate-adsorbent interactions or 'guest-host interactions' in supramolecular chemistry terminology. This is expected for TDA and TEO since they are lipophilic hosts without hydrogen bond donor groups (Fig. 2). When the host has a more hydrophilic character, such as is the case of the TBA with its hydroxybiphenyl arms, the water has more sites to hydrogen bond with, leading to a water condensation phenomenon below its saturation pressure at $298 \mathrm{~K}$ (Fig. 6). The total water uptake for TBA (approx. $4.3 \mathrm{mmol} / \mathrm{g}$ at $\mathrm{p} / \mathrm{p}_{0}=0.95$ ) was ten times higher than in the case of TDA or TEO. So, TBA showed the only noteworthy uptake, most probably due to hydrogen bonding interactions. In the context of hydrophobicity, the $\log \mathrm{P}$ values of all three hosts were calculated via Molinspiration to be $\log \mathrm{P}$ TDA $=8.30, \log \mathrm{P} \mathrm{TEO}=9.47$ and $\log \mathrm{P}$ TBA $=9.86$.

Next, THF adsorption/desorption measurements were performed in order to evaluate a less polar solvent. The shape of the isotherms for the TBA and TEO at $298 \mathrm{~K}$ (Fig. 7a) are type II according to the IUPAC classification (Thommes et al. 2015), indicating significant interactions between guest and host, whereas TDA shows type III shape (weak interaction). Condensation just below the saturation pressure was detected in all cases. The total uptake at $p /$

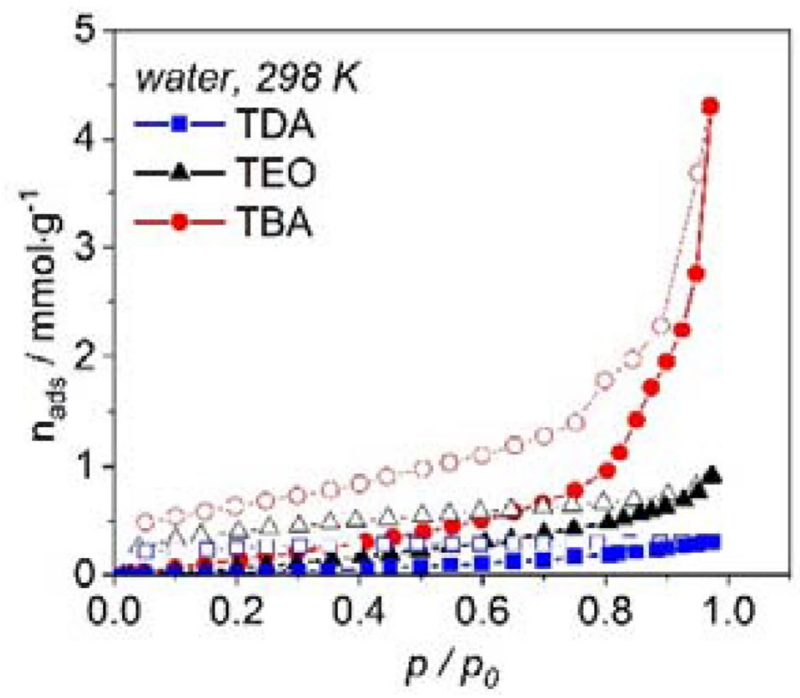

Fig. 6 Adsorption (closed symbols) and desorption (open symbols) isotherms for water and the three TAAs tested, measured at a temperature of $298 \mathrm{~K}$ (Color figure online) 
(a)

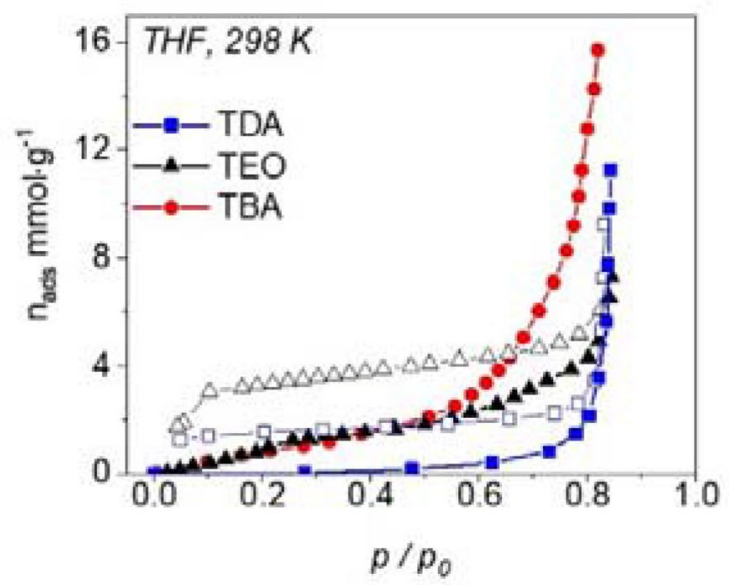

(b)

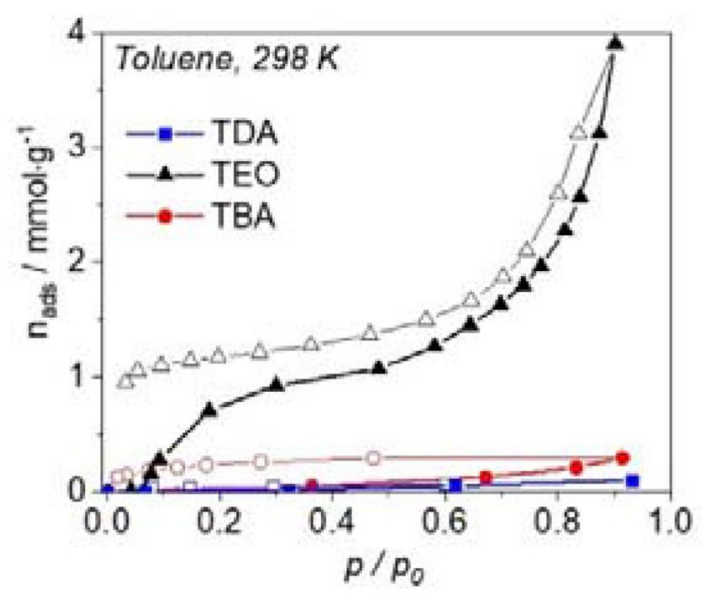

Fig. 7 Adsorption (closed symbols) and desorption (open symbols) isotherms at $298 \mathrm{~K}$ for: a THF at $298 \mathrm{~K}$, and b toluene and 1-3 as hosts (Color figure online)

$p_{0} \sim 0.8$ was ca. 11,7 and $14 \mathrm{~cm}^{3} / \mathrm{g}$ for TDA, TEO and TBA, respectively. So, both TBA and TEO showed considerable uptake at lower pressure, indicating incorporation into the material. Secondly, all of the isotherms show a hysteresis, probably caused by uptake with structural changes in the host material that slow down the uptake/release compared to simple adsorption/desorption.

Subsequently, we measured the adsorption/desorption isotherms using toluene as aromatic, non-polar guest. In this case, TDA and TBA showed weak interactions with the guest (type III isotherm), while TEO showed a clear type II isotherm. Interestingly, TEO does not show adsorption at very low relative pressure $\left(p / p_{0}<0.08\right)$, but a sudden uptake increase, leading to a concave curve up to $p / p_{0} \sim 0.4$ (Fig. 7b), where condensation commences. The total uptake for TEO is approx. $3.9 \mathrm{mmol} / \mathrm{g}$, i.e. more than ten times higher than in the case of TDA or TBA. The TAAs crystals were inspected under the optical microscope before and after physisorption experiments. The crystal morphology remained the same, although the surface of crystals looked slightly different after ad-/desorption in some cases, which points to reversibility of the host-guest interaction (see ESM, Fig. S1).

Since TAAs have no significant porosity, the magnitude of the uptake, especially at low relative pressure for the system TEO/toluene, cannot be based exclusively on adsorption on the external surface of the particles, but should be the result of an encapsulation of the guest into the crystal matrix, possibly leading to structures observed in the singlecrystal analysis (Fig. 3c). Indeed, the inclusion compounds can be prepared not only by recrystallization but also by interaction of the solid host with guest vapour, as it may be the case here (Schneider et al. 2012; Soldatov et al. 1999;
Soldatov and Ripmeester 2001; Nossov et al. 2001). Taken together, the results from the measurements with argon, THF and toluene showed that the polarity of the guest and host plays an important role for uptake. For TEO, the most nonpolar host, uptake of toluene is very significant, confirming that this TAA is particularly well suited for accommodating BTX aromatics (Schwenger et al. 2017).

We then evaluated the capability of TAA crystals to take up methane. The uptake experiments were performed under two different settings: vacuum-dried crystals or crystals pretreated with water to allow for the formation of methane hydrates. Methane hydrate (MH) is an ice-like compound that is formed when water and methane come into contact under thermodynamically favorable conditions (Koh et al. 2011). The hydrate is not easy to prepare artificially due to the slow kinetics of formation, which are on the time scale of weeks. There are currently more and more studies on formation of methane hydrate in porous materials. Several approaches have been tested to increase the gas-liquid contact area, and consequently the rate of $\mathrm{MH}$ formation (Linga et al. 2009; Mel'nikov et al. 2016; Park and Kim 2013). Nanoporous materials have proven to be an excellent platform for hosting and promoting the formation of MHs in a matter of minutes (Borchardt et al. 2018; Casco et al. 2019). Still, there is little known on how surfaces can be used to facilitate $\mathrm{MH}$ growth. Moreover, until now, only inorganic materials such as zeolites, MOFs, carbons, and silica have been used successfully to promote $\mathrm{MH}$ formation (Linga et al. 2009).

First, the vacuum-dried crystals were employed in our experiments with methane. Specifically, high-pressure methane adsorption/desorption experiments were conducted at 243 and $275 \mathrm{~K}$ for the three TAAs up to a pressure of $6 \mathrm{MPa}$ 

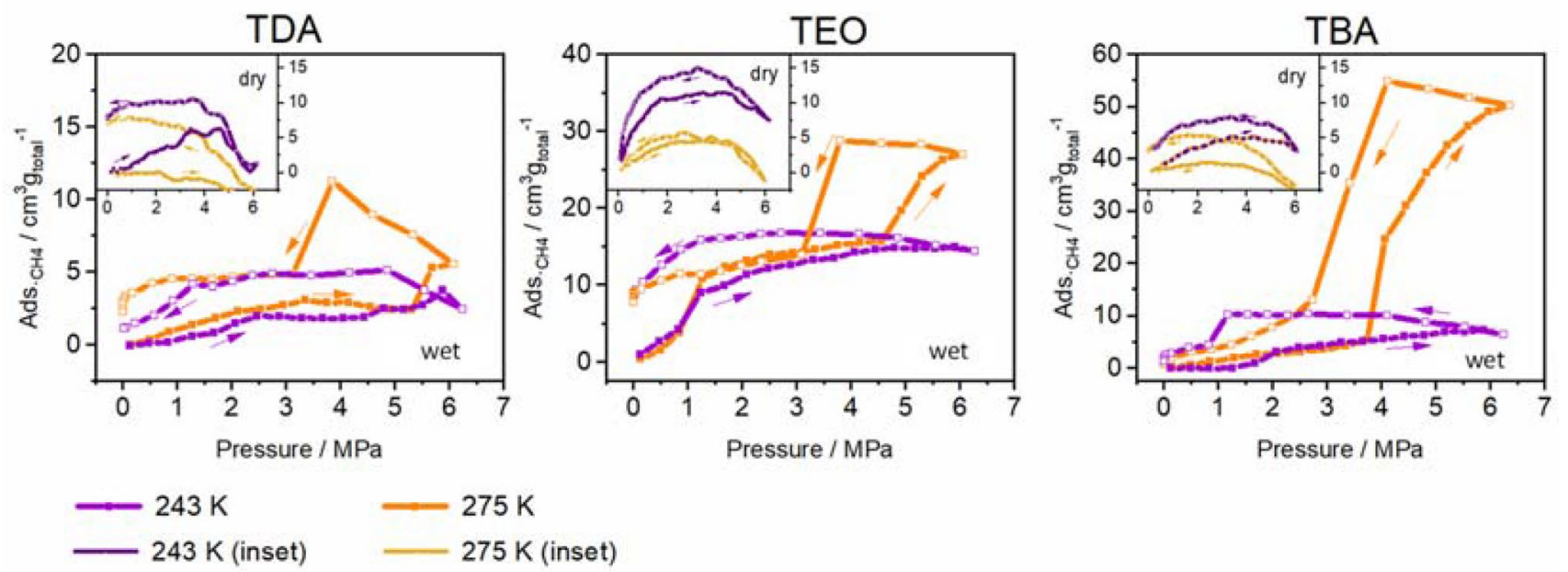

Fig. 8 Wet TAA crystals take up methane at $275 \mathrm{~K}$. Methane adsorption (closed-symbols) and desorption (empty-symbols) isotherms at $243 \mathrm{~K}$ (violet) and $275 \mathrm{~K}$ (orange) up to $6 \mathrm{MPa}$ for TDA, TEO and TBA pre-humidified with a mass ratio of $1: 1$. The $y$-axes give $\mathrm{cm}^{3}$ of methane per $g$ of TAA. Insets: Results of the same experiments with-

(insets in Fig. 8). At $275 \mathrm{~K}$, gas uptake values were found to be small, as expected for organic crystals without extrinsic or intrinsic porosity (Casco et al. 2015a, b). The best performance was observed for TEO, which gave values up to approx. $15 \mathrm{~cm}^{3} / \mathrm{g}$ at $243 \mathrm{~K}$ and $3 \mathrm{MPa}$. This is approx. one molar equivalent of methane per TAA that is taken up in dry form.

The sorption behaviour changed significantly when water was added. To conduct the experiments, TAAs were pre-impregnated with water up to a mass ratio of $1: 1$. The adsorption and desorption branches of the volumetrically measured isotherms (main plots of Fig. 8) exhibit a very different shape than the one expected for a pure physisorption phenomenon.

The maximal adsorption capacities at $243 \mathrm{~K}$ were small in all cases $\left(5-10 \mathrm{~cm}^{3} / \mathrm{g}\right)$. They are comparable to those in the dry state. At this low temperature, the water is in the solid state. An intimate contact of methane with the surface of the TAA crystals may be critical to initiate fast $\mathrm{MH}$ formation, which was difficult to achieve through the solid. Consequently, a low value of methane uptake due to $\mathrm{MH}$ formation was observed under these conditions.

This changed when the temperature was increased. Methane uptake was greatly improved at $275 \mathrm{~K}$ in the presence of water. Above $4 \mathrm{MPa}$, a second transition, associated with significant hysteresis, can be discerned under these conditions (Fig. 8), i.e. at a temperature at which water is in the liquid state. Methane total uptake values of $10,30,55 \mathrm{~cm}^{3} / \mathrm{g}$ were measured at $275 \mathrm{~K}$ and $6 \mathrm{MPa}$ for TDA, TEO and TBA, respectively.

We assign the steep increase above $4 \mathrm{MPa}$ to the formation of methane hydrate (Linga et al. 2009; Casco et al. out addition of water: Methane adsorption (closed symbols) and desorption (open symbols) isotherms at $243 \mathrm{~K}$ (dark violet) and $275 \mathrm{~K}$ (light orange) up to $6 \mathrm{MPa}$ for dry TDA, TEO and TBA (Color figure online)

2015a, b; Uchida et al. 1999). The sudden increase in the adsorption branch at around $4-5 \mathrm{MPa}$ and $275 \mathrm{~K}$ is on the timescale of minutes/hours, especially for TBA. So, the phenomenon cannot be due to physisorption only. Most likely, the TAAs are able to promote nucleation and formation of MHs. The ability to take up methane depends on the structure of the TAA. Probably, the presence of the hydroxybiphenyl arms in TBA make the crystals of this compound more hydrophilic (see Fig. 4), giving TBA the ability to "spread" water molecules along surfaces, thus producing a favourable methane/water interface for MH formation. We note that the theoretical unit cell diameter of $\mathrm{MH}$ is about $1.2 \mathrm{~nm}$ (Linga et al. 2009), i.e. too big to be incorporated into the TAA crystal structure. It is therefore reasonable to assume that MH forms on the outer surface of the TAA crystals. Similar conclusion was published by Andres-García et al. for microporous zeolite (Andrés-García et al. 2019).

The high-pressure methane adsorption isotherms for wet TEO (2) are consistent with the hypothesis that approx. one molar equivalent is taken up into the crystals themselves (up to a pressure of $1 \mathrm{MPa}$ ) and that the uptake of much larger quantities is induced via methane hydrate formation, probably outside the crystal lattice of the TAA. The onset of MH-based uptake occurs above $3 \mathrm{MPa}$, as expected based on the phase diagram for MHs. Here, in the presence of water, facilitated methane hydrate growth picks up while the ad/absorption sites within the TAA crystals probably reach saturation.

The extent to which the MH-based mechanism operates is different for the three TAAs. For TDA (1), which shows minimal affinity for water, even when approaching a value of 1 for the partial pressure in the sorption experiment (Fig. 6), 
the ability to induce MH formation is smallest. For TEO (2), it is more pronounced, and for TBA (3), with its hydroxy groups at the distal end of the biphenyl arms and an accessible hydrate structure (Fig. 4), it is greatest. So, the surfaces of the TBA crystals are probably the most hydrophilic ones among the TAAs, favouring $\mathrm{MH}$ formation. In addition, the more open structure of TBA comparing to TEO and TDA, may contribute to increase the gas/liquid interface and promote $\mathrm{MH}$ formation. The unusual temperature dependence (negligible at $243 \mathrm{~K}$, significant at $275 \mathrm{~K}$ ) indicates that there are kinetic phenomena blocking uptake in the cold, but allowing for it above the freezing point of water. That the crystals are important in the phenomenon can be seen for the differences in the isotherms for compounds $\mathbf{1 - 3}$, including the more pronounced hysteresis in the case of $\mathbf{1}$ versus $\mathbf{2}$ and 3. Hydrophobicity alone is not sufficient to explain the differences; the ability to take up small quantities of methane into the crystals, as detected most pronounced for TEO may contribute to it.

High-pressure adsorption experiments under wet conditions are very sensitive to the kind of instrument used to measure the adsorption/desorption isotherms (gravimetric vs. volumetric), mainly because of their different versatility to set the equilibrium time. Moreover, the temperature used during the experiment determines the inflexion pressure at which the MH crystals form. Results from control experiments with either type of technique are presented in the ESM for TEO at $264 \mathrm{~K}$ (Fig. S2). Although the shape of the isotherm differs from Fig. 8, still shows the abrupt step, which in the literature is attributed to the formation of methane hydrate at the pressure of around $3 \mathrm{MPa}$. To confirm the formation of methane hydrate crystals on TEO, we then conducted exploratory synchrotron PXRD measurements at increasing pressures of methane with wet sample at $264 \mathrm{~K}$. It is important to mention that the wet TEO was exposed to methane gas for two hours per pressure step before recording the diffractograms (Fig. 9). in order to give the system enough time to reach the equilibrium The results revealed, firstly, a mixture of cubic and hexagonal ice pattern at a pressure of $0.1 \mathrm{MPa}$. Secondly, we saw additional peaks occurring at elevated gas pressures. These peaks correspond to the methane hydrate structure I $(s I)$. No changes in the TEO structure is detected during the experiment which suggest that $\mathrm{MH}$ crystals form over the surface.

\section{Conclusions}

In summary, adsorption/desorption experiments with tetraaryladamantanes known to encapsulate guest molecules upon crystallization, but without significant porosity, were shown

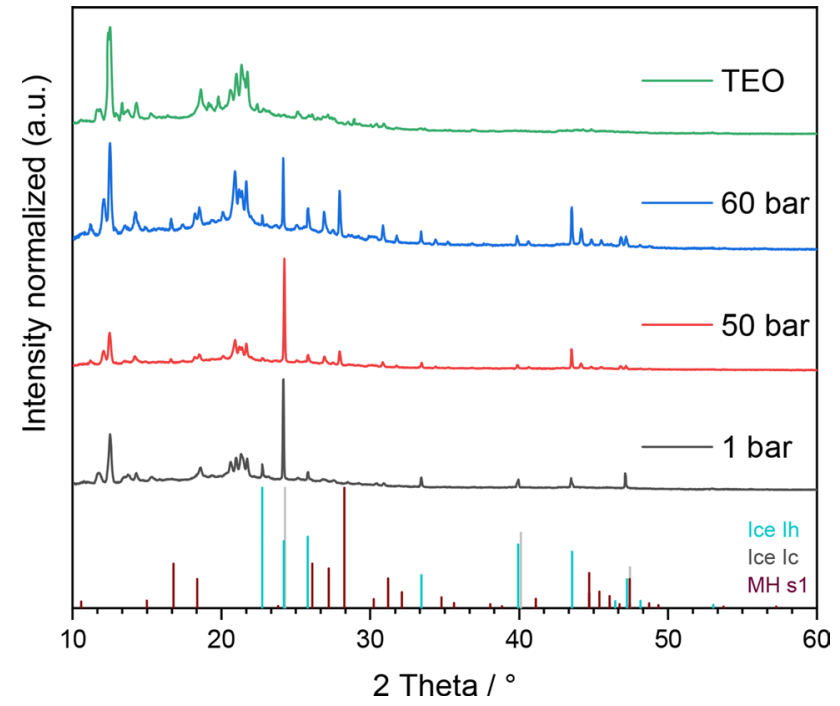

Fig. 9 In situ synchrotron PXRD of wet TEO (mass ratio 1:1) at methane pressures of $0.1,5$ and $6 \mathrm{MPa}$ at $264 \mathrm{~K}$. The PXRD patterns of MH structure sI, ice Ih, ice Ic and dry TEO are included for the sake of comparison

to take up different molecules from the gas phase such as water, methane, THF and toluene. Up to approx. one equivalent of methane was taken up in the dry state. Rather than relying on "porosity without pores"(Barbour 2006), the crystals of the tetraaryladamantanes probably possess low energy barriers for structural rearrangements that allow for this. Moreover, the TAA crystals lead to an uptake of up to $55 \mathrm{~cm}^{3} / \mathrm{g}$ methane at $275 \mathrm{~K}$ and $6 \mathrm{MPa}$ for wet TBA, most likely via facilitated formation of methane hydrates, if impregnated with water. SPXRD patterns confirm MH sI for wet TEO in concordance with the ad-/desorption experiments. Our findings thus add a fascinating facet to a class of compounds with an unusual breadth of crystalline states. The uptake of an industrially relevant gas into materials that are easy to produce from organic solutions and free of heavy elements may also lead to practical applications.

Acknowledgements This work was supported by DFG (Grant No. RI 1063/15-1 to C.R.) and the University of Stuttgart. MEC thanks the Alexander von Humboldt foundation and Agencia Nacional de Investigación e Innovación (PD_NAC_2018_1_150145) for financial support, LB thanks the Daimler und Benz foundation for financial support; LB and SG gratefully acknowledge the Federal Ministry of Education and Research (Bundesministerium für Bildung und Forschung, BMBF) for support (Project Mechanocarb, Grant Number 03SF0498).

Author contributions All authors contributed to the study conception and design. Material synthesis and single XRD measurements were performed by FK. Adsorption isotherms of samples were measured by MEC. SXRD measurements were performed by SG. The manuscript was written by MEC and FK. All authors read and approved the final manuscript. 
Funding Open Access funding provided by Projekt DEAL.

\section{Compliance with ethical standards}

Conflict of interest The authors declare that they have no conflict of interest.

Open Access This article is licensed under a Creative Commons Attribution 4.0 International License, which permits use, sharing, adaptation, distribution and reproduction in any medium or format, as long as you give appropriate credit to the original author(s) and the source, provide a link to the Creative Commons licence, and indicate if changes were made. The images or other third party material in this article are included in the article's Creative Commons licence, unless indicated otherwise in a credit line to the material. If material is not included in the article's Creative Commons licence and your intended use is not permitted by statutory regulation or exceeds the permitted use, you will need to obtain permission directly from the copyright holder. To view a copy of this licence, visit http://creativecommons.org/licenses/by/4.0/.

\section{References}

Alexandre, P.-E., Schwenger, A., Frey, W., Richert, C.: High loading crystals of tetraaryladamantanes and the uptake and release of aromatic hydrocarbons from the gas phase. Chem. Eur. J. 23(38), 9018-9021 (2017)

Ananchenko, G.S., Moudrakovski, I.L., Coleman, A.W., Ripmeester, J.A.: A channel-free soft-walled capsular calixarene solid for gas adsorption. Angew. Chem. Int. Ed. 47(30), 5616-5618 (2008)

Andrés-García, A., Dikhtiarenko, A., Fauth, F., Silvestre-Albero, J., Ramos-Fernández, E.V., Gascón, J., Corma, A., Kapteijn, F.: Methane hydrates: nucleation in microporous materials. Chem. Eng. J. 360, 569-576 (2019)

Atwood, J.L., Barbour, L.J., Jerga, A.: Storage of methane and freon by interstitial van der Waals confinement. Science 296(5577), 2367-2369 (2002)

Atwood, J.L., Barbour, L.J., Jerga, A.: A new type of material for the recovery of hydrogen from gas mixtures. Angew. Chem. Int. Ed. 43(22), 2948-2950 (2004)

Baker, W., Floyd, A.J., McOmie, J.F.W., Pope, G., Weaving, A.S., Wild, J.H.: Condensation products of phenols and ketones. Part $\mathrm{X}$. The structure of dianin's compounds, a unique inclusionforming substance. J. Chem. Soc. (1956)

Barbour, L.: Crystal porosity and the burden of proof. Chem. Commun. 11, 1163-1168 (2006)

Borchardt, L., Casco, M.E., Silvestre-Albero, J.: Methane hydrate in confined spaces: an alternative storage system. ChemPhysChem. 19(11), 1298-1314 (2018)

Brouwer, D.H., Moudraskovski, I.L., Udachin, K.A., Enright, G.D., Ripmeester, J.A.: Guest loading and multiple phases in single crystals of the van der Waals host p-tert-butylcalix[4]arene. Cryst. Growth Des. 8(6), 1878-1885 (2008)

Casco, M.E., Martínez-Escandell, M., Gadea-Ramos, E., Kaneko, K., Silvestre-Albero, J., Rodríguez-Reinoso, F.: High-pressure methane storage in porous materials: are carbon materials in the pole position? Chem. Mater. 27(3), 959-964 (2015a)

Casco, M.E., Silvestre-Albero, J., Ramírez-Cuesta, A.J., Rey, F., Jordá, J., Bansode, L.A., Urakawa, A., Peral, I., MartínezEscandell, M., Kaneko, K., Rodríguez-Reinoso, F.: Methane hydrate formation in confined nanospace can surpass nature. Nat. Commun. 6, 6432-6440 (2015b)
Casco, M.E., Zhang, E., Grätz, S., Krause, S., Bon, V., Wallacher, D., Grimm, N., Többens, D.M., Hauß, T., Borchardt, L.: Experimental evidence of confined methane hydrate in hydrophilic and hydrophobic model carbons. J. Phys. Chem. C .123(39), 24071-24079 (2019)

Chaoui, N., Trunk, M., Dawson, R., Schmidt, J., Thomas, A.: Trends and challenges for microporous polymers. Chem. Soc. Rev. 46(11), 3302-3321 (2017)

Desiraju, G.R., Vittal, J.J.: Crystal engineering: a textbook. World Scientific, Singapore (2011)

Dianin, A.P.: On the condensation of phenols with unsaturated ketones. Condensations of phenols with mesitylene oxide. J. Soc. Phys. Chem. Russe 46, 1310-1319 (1914)

Enright, G.D., Udachin, K.A., Moudrakovski, I.L., Ripmeester, J.A.: Thermally programmable gas storage and release in single crystals of an organic van der Waals host. J. Am. Chem. Soc. 125(33), 9896-9897 (2003)

Gu, C., Hosono, N., Zheng, J.-J., Sato, Y., Kusaka, S., Sakaki, S., Kitagawa, S.: Design and control of gas diffusion process in a nanoporous soft crystal. Science 363(6424), 387-391 (2019)

Holst, J.R., Trewin, A., Cooper, A.I.: Porous organic molecules. Nat. Chem. 2, 915-920 (2010)

Koh, C.A., Sloan, E.D., Sum, A.K., Wu, D.T.: Fundamentals and applications of gas hydrates. Annu. Rev. Chem. Biomol. Eng. 2, 237-257 (2011)

Krupp, F., He, S., Frey, W., Richert, C.: A crystalline ready-to-use form of cyclopentadiene. Synlett 29(13), 1707-1710 (2018)

Linga, P., Haligva, C., Nam, S.C., Ripmeester, J.A., Englezos, E.: Gas hydrate formation in a variable volume bed of silica sand particles. Energy Fuels. 23(11), 5496-5507 (2009)

Mel'nikov, V.P., Podenko, L.S., Nesterov, A.N., Drachuk, A.O., Molokitina, N.S., Reshetnikov, A.M.: Self-preservation of methane hydrates produced in "dry water". Dokl. Chem. 466, 53-56 (2016)

Nossov, A.V., Soldatov, D.V., Ripmeester, J.A.: In situ switching of sorbent functionality as monitored with hyperpolarized ${ }^{129} \mathrm{Xe}$ NMR spectroscopy. J. Am. Chem. Soc. 123(15), 3563-3568 (2001)

Park, S.-S., Kim, N.-J.: Study on methane hydrate formation using ultrasonic waves. J. Ind. Eng. Chem. 19(5), 1668-1672 (2013)

Richert, C., Krupp, F.: Encapsulating organic crystals (EnOCs). Synlett 28(14), 1763-1766 (2017)

Schilling, C., Plietzsch, O., Nieger, M., Muller, T., Bräse, S.: Fourfold Suzuki-Miyaura and Sonogashira cross-coupling reactions on tetrahedral methane and adamantane derivatives. Eur. J. Org. Chem. 9, 1743-1754 (2011)

Schneider, M.W., Oppel, I.M., Ott, H., Lechner, L.G., Hauswald, H.-jS., Stoll, R., Mastalerz, M.: Periphery-substituted [4 + 6] salicylbisimine cage compounds with exceptional high surface areas: influence of the molecular structure on nitrogen sorption properties. Chem. Eur. J. 18(3), 836-847 (2012)

Schüth, F., Sing, K.S.W., Weitkamp, J.: Handbook of Porous Solids. Wiley-VCH Verlag GmbH, Weinheim (2002)

Schwenger, A., Frey, W., Richert, C.: Tetrakis(dimethoxyphenyl) adamantine (TDA) and its inclusion complexes in the crystalline state: a versatile carrier for small molecules. Chem. Eur. J. 21(24), 8781-8789 (2015)

Schwenger, A., Birchall, N., Richert, C.: Reagents with a crystalline coat. Angew. Chem. Int. Ed. 55(44), 13706-13709 (2016)

Schwenger, A., Birchall, N., Richert, C.: Solution-phase synthesis of branched oligonucleotides with up to 32 nucleotides and the reversible formation of materials. Eur. J. Org. Chem. 39, 58525864 (2017)

Singh, A., Tolev, M., Meng, M., Klenin, K., Plietzsch, O., Schilling, C.I., Muller, T., Nieger, M., Braese, S., Wenzel, W., Richert, C.: 
Branched DNA that forms a solid at $95{ }^{\circ} \mathrm{C}$. Angew. Chem. Int. Ed. 50(14), 3227-3231 (2011)

Singh, A., Tolev, M., Schilling, C.I., Bräse, S., Griesser, H., Richert, C.: Solution-phase synthesis of branched DNA hybrids via H-Phosphonate dimers. J. Org. Chem. 77(6), 2718-2728 (2012)

Soldatov, D.V., Ripmeester, J.A.: Novel 4-vinylpyridine-extended metal-dibenzoylmethanate host frameworks: structure, polymorphism, and inclusion properties. Chem. Eur. J. 7(14), 2979-2994 (2001)

Soldatov, D.V., Ripmeester, J.A., Shergina, S.I., Sokolov, I.E., Zanina, A.S., Gromilov, S.A., Dyadin, Y.A.: $\alpha$-and $\beta$-Bis(1,1,1-trifluoro-5, 5-dimethyl-5-methoxyacetylacetonato) copper (II): Transforming the dense polymorph into a versatile new microporous framework. J. Am. Chem. Soc. 121(17), 4179-4188 (1999)

Sozzani, P., Bracco, S., Comotti, A., Ferreti, L., Simonutti, R.: Methane and carbon dioxide storage in a porous van der Waals crystal. Angew. Chem. Int. Ed. 44(12), 1816-1820 (2005)

Thallapally, P.K., Wirsig, T.B., Barbour, L.J., Atwood, J.L.: Crystal engineering of nonporous organic solids for methane sorption. Chem. Commun. 35, 4420-4422 (2005)

Thallapally, P.K., Kirby, K.A., Atwood, J.L.: Comparison of porous and nonporous materials for methane storage. New J. Chem. 31(5), $628-630$ (2007)
Thommes, M., Kaneko, K., Neimark, A.V., Olivier, J.P., RodriguezReinoso, F., Rouquerol, J., Sing, K.S.W.: Physisorption of gases, with special reference to the evaluation of surface area and pore size distribution (IUPAC Technical Report). Pure Appl. Chem 87(9-10), 1051-1069 (2015)

Tian, J., Thallapally, P.K., Dalgarno, S.J., Atwood, J.L.: Free transport of water and $\mathrm{CO}_{2}$ in nonporous hydrophobic clarithromycin form II crystals. J. Am. Chem. Soc. 131(37), 13216-13217 (2009)

Tian, J., Thallapally, P.K., McGrail, B.P.: Porous organic molecular materials. Cryst. Eng. Comm. 14(6), 1909-1919 (2012)

Uchida, T., Ebinuma, T., Ishizaki, T.: Dissociation condition measurements of methane hydrate in confined small pores of porous glass. J. Phys. Chem. B. 103(18), 3659-3662 (1999)

Zhang, G., Presly, O., White, F., Oppel, I.M., Mastalerz, M.: A permanent mesoporous organic cage with exceptionally high surface area. Angew. Chem. Int. Ed. 53(6), 1516-1520 (2014)

Zhou, H.-C., Long, J.R., Yaghi, O.M.: Introduction to metal-organic frameworks. Chem. Rev. 112, 673-674 (2012)

Publisher's Note Springer Nature remains neutral with regard to jurisdictional claims in published maps and institutional affiliations.

\section{Affiliations}

\section{Mirian Elizabeth Casco ${ }^{1}$ - Felix Krupp ${ }^{2}$ - Sven Grätz ${ }^{3}$ - Alexander Schwenger ${ }^{2}$. Vassiliki Damakoudi ${ }^{2}$. Clemens Richert ${ }^{2} \cdot$ Wolfgang Frey $^{2} \cdot$ Lars Borchardt $^{3}$ (D)}

Lars Borchardt

lars.borchardt@rub.de

1 Department of Engineering, Catholic University of Uruguay, Av. 8 de Octubre 2738, 1160 Montevideo, Uruguay
2 Institute of Organic Chemistry, University of Stuttgart, 70569 Stuttgart, Germany

3 Inorganic Chemistry, Ruhr-University Bochum, 44801 Bochum, Germany 